\title{
Course of respiratory allergy by treatment strategy based on German routine data
}

\author{
Ann-Kathrin Weschenfelder · Ludger Klimek · Norbert Mülleneisen · Harald Renz • Wolfgang Wehrmann • \\ Thomas Werfel · Eckard Hamelmann · Jürgen Wasem · Janine Biermann
}

Received: 10 February 2017 / Accepted: 2 May 2017 / Published online: 30 May 2017

(C) The Author(s) 2017. This article is an open access publication.

\begin{abstract}
Purpose Allergic respiratory diseases represent a global health problem. The two major treatment strategies are symptom treatment and specific immunotherapy (SIT). SIT is considered the only causal treatment option available with the ability to alter the course of the disease. This study aims to describe the course of disease and medication of respiratory allergy across treatment strategies and disease groups.
\end{abstract}

This study was conducted at the Institute for Health Care Management and Research at the University of Duisburg-Essen, Germany

A.-K. Weschenfelder, M.Sc. · Prof. J. Wasem · Dr. J. Biermann Institute for Health Care Management and Research, University of Duisburg-Essen, Essen, Germany

A.-K. Weschenfelder, M.Sc. $(\bowtie)$

Thea-Leymann-Str. 9, 45127 Essen, Germany

Ann-Kathrin.Weschenfelder@medman.uni-due.de

Prof. L. Klimek

Centre for Rhinology and Allergology, Wiesbaden, Germany

N. Mülleneisen

Asthma and Allergy Centre, Leverkusen, Germany

Prof. H. Renz

Institute of Laboratory Medicine and Pathobiochemistry, Molecular Diagnostics, Philipps University Marburg,

Marburg, Germany

Prof. W. Wehrmann

Dermatological Clinic Prof. Wehrmann, Dr.

Rödder-Wehrmann and Colleagues, Münster, Germany

Prof. T. Werfel

Division of Immunodermatology and Allergy Research, Department of Dermatology and Allergy, Hannover Medical School, Hannover, Germany

Prof. E. Hamelmann

Children's Center Bethel, Protestant Hospital Bielefeld and Allergy Center, Ruhr-University, Bochum, Germany
Methods The analysis is based on routine data from a German statutory health insurance. The patient cohort is observed from 2007-2012. For each year based on assured outpatient diagnoses patients are assigned to a disease group: rhinitis, asthma or both diseases. Additionally, prescribed medication is considered. Treatment comparisons are based on matched pairs. Results The study population comprises 165,446 patients with respiratory allergy. In 2007 the most frequent disease group is rhinitis $(70 \%)$, followed by asthma (16\%) and both diseases (14\%). During the observation period a second allergic respiratory diagnosis occurs only in about $12 \%$ of rhinitis patients and $28 \%$ of asthma patients. In about $50 \%$ of patients with both diseases one of the diagnoses is omitted. These patients are more likely to no longer report their asthma diagnosis when receiving immunotherapy compared to symptom treatment. Furthermore immunotherapy reduces the frequency of asthma medication use.

Conclusions Results of detailed analysis of diagnoses reflect the alternating nature of allergic diseases. Although limited by accuracy of documentation and the lack of clinical information, the comparison of treatment strategies shows some advantages of immunotherapy regarding course of disease and asthma medication use.

Keywords Disease progression · Drug therapy · Desensitization, immunologic · Sublingual immunotherapy · Rhinitis, allergic $\cdot$ Asthma

$\begin{array}{ll}\text { Abbreviations } \\ \text { AA Allergic asthma } \\ \text { AR } & \text { Allergic rhinitis } \\ \text { ARAA } & \text { Allergic rhinitis with concomitant allergic } \\ & \text { asthma } \\ \text { ATC } & \text { Anatomical Therapeutic Chemical }\end{array}$


ICD International Classification of Diseases

mST Matched symptom treatment

OR Odds ratio

SCIT Subcutaneous immunotherapy

SHI Statutory health insurance

SIT Specific immunotherapy

SLIT Sublingual immunotherapy

ST Symptom treatment

GM German modification

\section{Introduction}

Occurring in both developed and developing countries and across all ethnic groups and ages allergic respiratory diseases represent a global health problem [1]. Their global prevalence ranges between $5 \%$ and $40 \%$ [2, 3]; in Germany the prevalence for asthma is $9 \%$ and for allergic rhinitis it is $15 \%$ [4]. Respiratory allergy patients experience symptoms and impairments in daily life activities, social life, sleep and ability to work reducing their quality of life [5-7].

Specific immunotherapy (SIT) is able to influence the underlying immunological mechanisms of allergy [8]. It can be applied as subcutaneous injection (SCIT) or sublingual treatment (SLIT) [9]. Additionally, various pharmaceuticals are available to treat symptoms of allergic respiratory diseases [1, 10]. While allergen avoidance can be seen as further treatment strategy it is often not completely feasible in daily life. Therefore, specific immunotherapy is considered the only treatment for respiratory allergy with the ability to alter the natural course of the disease [8].

This study aims to first describe the course of allergic respiratory diseases under different treatment strategies and, second to compare the course of disease and the use of prescription drugs between the treatment strategies.

\section{Materials and methods}

This study is based on routine data of a nationwide German statutory health insurance (SHI) company available for the years 2005-2012. The study population is derived from all insured persons in 2007. This cohort is then observed from the baseline year 2007 until 2012, while 2005 and 2006 data are used for population selection.

Diagnoses are available as International Classification of Diseases (ICD) codes version 10 German modification (GM). Patients are considered to have allergic respiratory disease when they received at least one assured outpatient diagnosis of J30.1 "allergic rhinitis due to pollen", J30.2 "other seasonal allergic rhinitis", J30.3 "other allergic rhinitis" or J30.4 "allergic rhinitis, unspecified" for allergic rhinitis or J45.0 "predominantly allergic asthma” or J45.8 "mixed asthma” for allergic asthma within the year under consideration $[11,12]$. The choice of these asthma ICD codes ensures an allergic component of the disease.
Based on their diagnoses insured persons are grouped to the following disease groups for each year: isolated allergic rhinitis (AR), isolated allergic asthma (AA) and allergic rhinitis with concomitant allergic asthma (ARAA). This third group of patients having both diseases is established to account for differences in patients' quality of life and treatment requirements. These differences result from the negative influence of having both diseases on the severity of each single disease [13, 14]. The course of disease is analyzed based on these three disease groups.

Disease-related prescription drugs are identified using the Anatomical Therapeutic Chemical (ATC) codes and their subordinated national drug codes. Relevant active ingredients are listed in Table 1. The analysis excludes over-the-counter drugs as these are only documented for insured persons under the age of 12. Based on their prescriptions, insured persons are assigned to the following medication groups for each year: no medication, asthma medication, rhinitis medication and medication for both diseases.

Treatment strategies under investigation are specific immunotherapy (SIT) with symptom treatment as needed and symptom treatment alone (ST). Within specific immunotherapy subcutaneous and sublingual application are distinguished. Immunotherapy prescriptions are identified through ATC codes and national drug codes for inhalant allergen extracts.

The study cohort is established based on 2007 data. Inclusion criteria are having an allergic respiratory disease diagnosis and data being available in 2005-2012. Exclusion criteria are age younger than five years and SIT prescription in 2005 or 2006 . Insured persons under the age of five are excluded because medical guidelines do not recommend the use of SIT below this age [15]. To avoid possible bias from recently conducted courses of SIT only insured persons starting immunotherapy are analyzed by excluding those with immunotherapy prescription in 2005 or 2006.

Table 1 Active ingredients for symptom treatment of allergic respiratory diseases relevant to the study

\begin{tabular}{|c|c|}
\hline ATC code & Active pharmaceutical ingredient group \\
\hline \multicolumn{2}{|l|}{ Rhinitis } \\
\hline R01AD & Topical corticosteroids \\
\hline \multicolumn{2}{|l|}{ Asthma } \\
\hline R03AA & Alpha- and beta-adrenoreceptor agonists ${ }^{\mathrm{a}}$ \\
\hline R03AC & Selective beta-2-adrenoreceptor agonists ${ }^{\mathrm{a}}$ \\
\hline R03AK & $\begin{array}{l}\text { Adrenergics in combination with corticosteroids or } \\
\text { other drugs, excluding anticholinergics }\end{array}$ \\
\hline R03BA & Glucocorticoids $^{\mathrm{a}}$ \\
\hline R03BB & Anticholinergics $^{\mathrm{a}}$ \\
\hline R03BC & Antiallergic agents, excl. corticosteroids ${ }^{a}$ \\
\hline R03DC03 & Montelukast \\
\hline R03DX05 & Omalizumab \\
\hline
\end{tabular}


Fig. 1 Population selection algorithm (SCIT subcutaneous immunotherapy, SLIT sublingual immunotherapy, ST symptom treatment, $m S T$ matched symptom treatment group)

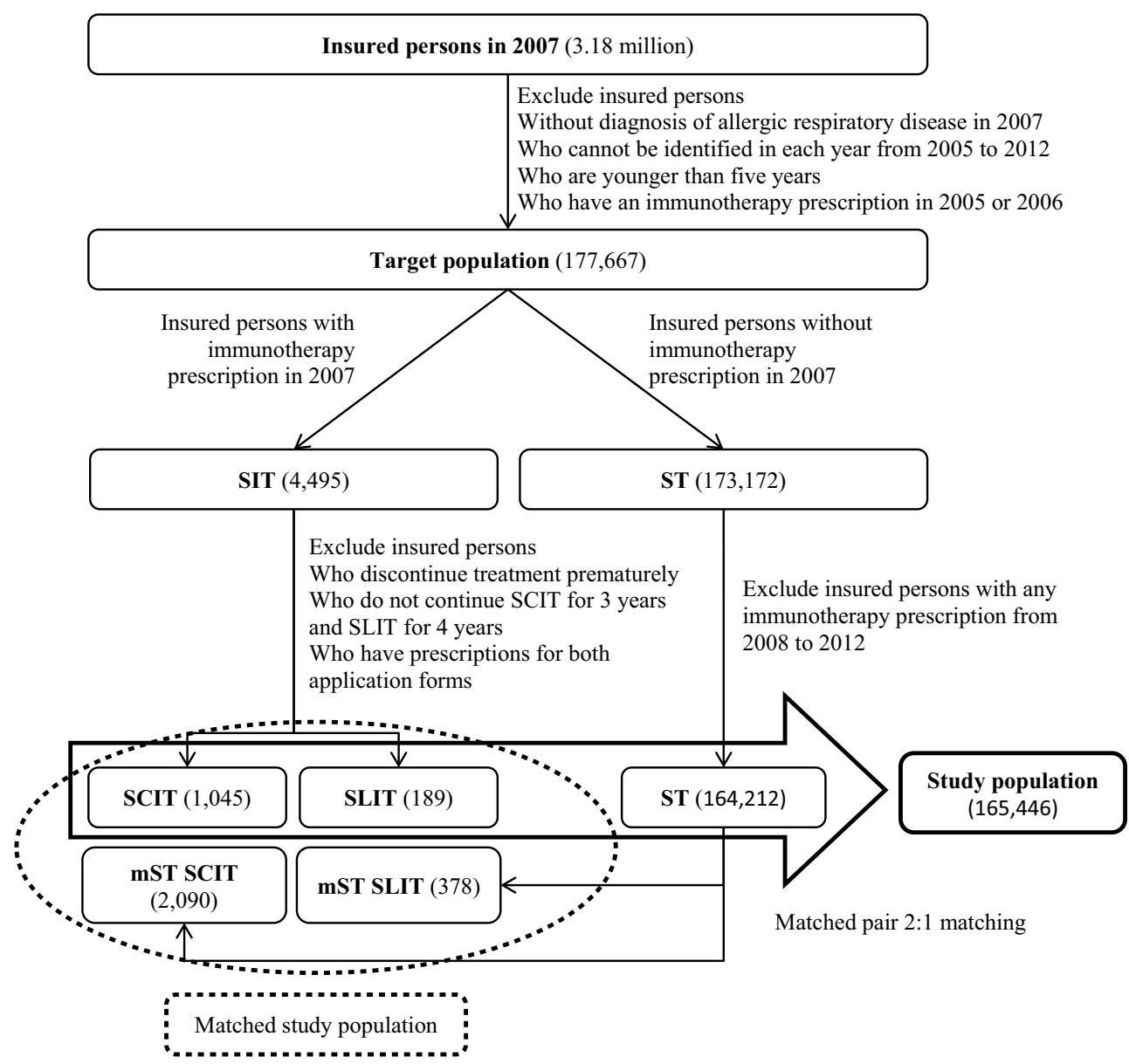

The selected population is split into SIT and ST treatment strategy based on their prescriptions in 2007. In the ST group a further exclusion criterion is having a SIT prescription during the observation period (2008-2012). By splitting the SIT group into SCIT and SLIT insured persons with prescriptions for both application forms are excluded. To achieve homogeneity of analyzed treatment phases within each type of SIT, only the most frequently recorded treatment duration is taken into account. Selection is based on reported treatment duration due to the lack of a definite recommendation in German medical guidelines [15]. Therefore insured persons are excluded if their treatment duration is shorter than the minimum requirement of three years and not equal to the most frequently reported duration.

To compare the course of disease and medication between the treatment strategies a matched study population is defined. Using an exact matched pair method without replacement each person in the SCIT and SLIT group is assigned to two persons of the symptom treatment group. The matching criteria are the following: five year age groups, gender, and baseline disease and medication group (Fig. 1 summarizes the population selection process).

Statistical analyses are conducted using IBM SPSS version 21.0. All group differences are tested at a sig- nificance level of $5 \%$ applying the $\mathrm{Chi}^{2}$-test except of the number of medication-free years which are assessed by Mann-Whitney U-test. In case of small numbers of observations leading to invalid $\mathrm{Chi}^{2}$-test results the Fishers Exact test is applied. Odds ratios are calculated using univariate logistic regression.

\section{Results}

\section{Population}

In 2007 a total of 177,667 insured persons (mean age $44 \pm 21$ years, $66 \%$ female) met the inclusion criteria. Of those about $2.5 \%$ started SIT in 2007 (2\% SCIT, $0.5 \%$ SLIT and $0.03 \%$ both application forms). Treatment discontinuation rate before the required minimum period of 3 years is $41 \%$ for SCIT and $52 \%$ for SLIT. Most discontinuations occur after the first year of treatment ( $64 \%$ SCIT, $73 \%$ SLIT); however, some of those resume therapy at a later time (27\% SCIT, $7 \%$ SLIT). The most frequently reported treatment duration is 3 years for SCIT ( $42 \%$ of adherent patients) and 4 years for SLIT (43\% of adherent patients).

After applying the treatment-group-specific exclusion criteria the study population comprises 165,446 insured persons (Table 2). The largest proportion of this population $(70 \%)$ is assigned to the disease group 
Table 2 Study population by disease group and treatment strategy

\begin{tabular}{ll|ll|l}
\hline Disease group & Study population & & & ST \\
\hline AR & SCIT & SLIT & 115,379 & 116,213 \\
\hline ARAA & 705 & 129 & 20,781 & 21,139 \\
\hline AA & 307 & 51 & 28,052 & 28,094 \\
\hline Total & 33 & 9 & 164,212 & 165,446 \\
\hline $\begin{array}{l}\text { AR allergic rhinitis, } A A \text { allergic asthma, } \\
\text { ST symptom treatment }\end{array}$ & ARAA allergic rhinitis and allergic asthma, SCIT subcutaneous immunotherapy, SLIT sublingual immunotherapy, \\
\hline
\end{tabular}

isolated rhinitis followed by isolated asthma (16\%) and both diseases (14\%).

The pool of eligible matching partners for the SCIT and SLIT groups contains the entire symptom treatment group ("ST" column in Table 2). The matching was successfully conducted resulting in matched symptom treatment (mST) groups twice the size of the SCIT and SLIT group.

\section{Course of disease across treatment strategies}

In patients with isolated rhinitis at baseline the most frequent courses of disease are omission of rhinitis diagnosis (34\%), continuous diagnosis of rhinitis (32\%) and intermittent rhinitis diagnosis and diagnosis-free years $(21 \%)$. Less than $20 \%$ of patients with rhinitis document asthma as additional diagnosis during the observation period. Of those the largest proportion shows both diseases concomitantly rather than periods of isolated asthma and isolated rhinitis (AR and AA) or periods of all three disease groups (AR, AA and ARAA) interchangeably (Table 3).

In the population with both diseases at baseline the most frequent courses of disease are both diagnoses continuously (31\%), omission of both diagnoses (13\%), omission of asthma diagnosis followed by continuous rhinitis diagnosis (12\%) and remission of rhinitis diagnosis followed by continuous asthma diagnosis (9\%). Furthermore patients report omission of asthma diagnosis followed by omission of the remaining rhinitis diagnosis $(7 \%)$ or reoccurrence of the concomitant asthma diagnosis (7\%). Another $6 \%$ of the population each show both diagnoses with diagnosis-free periods or omission and reoccurrence of rhinitis. About half of the population with both

Table 3 Five year course of disease of insured persons with isolated rhinitis $(A R)$ at baseline by treatment strategy

\begin{tabular}{|c|c|c|c|c|c|}
\hline Description & SCIT & mST SCIT & SLIT & mST SLIT & ST total \\
\hline$N$ total & $\begin{array}{l}705 \\
\text { (in \%) }\end{array}$ & $\begin{array}{l}1410 \\
\text { (in \%) }\end{array}$ & $\begin{array}{l}129 \\
\text { (in \%) }\end{array}$ & $\begin{array}{l}258 \\
\text { (in \%) }\end{array}$ & $\begin{array}{l}115,379 \\
\text { (in \%) }\end{array}$ \\
\hline Only AR diagnosis ${ }^{C}$ & 84 & 85 & 82 & 90 & 88 \\
\hline Continuous AR diagnosis ${ }^{\mathrm{a}, \mathrm{c}}$ & 32 & 27 & 34 & 27 & 32 \\
\hline Intermittent AR diagnosis ${ }^{\mathrm{a}, \mathrm{d}}$ & 15 & 22 & 13 & 22 & 21 \\
\hline Omission of AR diagnosis & 36 & 36 & 35 & 41 & 34 \\
\hline Additional AA diagnosis ${ }^{C}$ & 16 & 15 & 18 & 10 & 12 \\
\hline AR followed by ARAA diagnosis ${ }^{a}$ & 13 & 10 & 14 & 6 & 9 \\
\hline AR followed by ARAA and omission & 3 & 2 & 3 & 2 & 2 \\
\hline AR followed by ARAA with omission of AA & 4 & 3 & 5 & 1 & 3 \\
\hline Intermittent AR and ARAA & 1 & 1 & 0 & 1 & 1 \\
\hline AR followed by ARAA & 5 & 4 & 6 & 3 & 4 \\
\hline$A R$ and $A A$ diagnoses $b$ & 2 & 3 & 2 & 2 & 2 \\
\hline AR followed by AA and omission & 1 & 1 & 2 & 1 & 1 \\
\hline AR followed by AA and AR & 0.4 & 0.4 & 0 & 1 & 0.2 \\
\hline AR followed by AA & 1 & 1 & 0 & 0.4 & 1 \\
\hline$A R, A A$ and $A R A A$ diagnoses & 2 & 2 & 2 & 2 & 1 \\
\hline AR followed by AA and ARAA & 0.1 & 0.4 & 0 & 0.4 & 0.2 \\
\hline AR followed by ARAA and AA & 1 & 1 & 1 & 0 & 1 \\
\hline Intermittent diagnoses of AR, AA and ARAA & 1 & 1 & 2 & 1 & 1 \\
\hline \multicolumn{6}{|c|}{$\begin{array}{l}\text { AR allergic rhinitis, } A R A A \text { allergic rhinitis and allergic asthma, } A A \text { allergic asthma, SCIT subcutaneous immunotherapy, SLIT sublingual immunotherapy, } \\
m S T \text { matched symptom treatment group, ST symptom treatment } \\
\text { aSCIT vs. mST SCIT } p \leq 0.05 \\
\text { bSCIT vs. mST SCIT } p \leq 0.01 \\
\text { ' } \text { SLIT vs. mST SLIT } p \leq 0.05 \\
\text { 'SLIT vs. mST SLIT } p \leq 0.01\end{array}$} \\
\hline
\end{tabular}


Table 4 Five-year course of disease of insured persons with both diseases $(A R A A)$ at baseline by treatment strategy

\begin{tabular}{|c|c|c|c|c|c|}
\hline & & & & & \\
\hline Description & SCIT & mST SCIT & SLIT & mST SLIT & ST total \\
\hline$N$ total & $\begin{array}{l}307 \\
\text { (in \%) }\end{array}$ & $\begin{array}{l}614 \\
\text { (in \%) }\end{array}$ & $\begin{array}{l}51 \\
\text { (in \%) }\end{array}$ & $\begin{array}{l}102 \\
\text { (in \%) }\end{array}$ & $\begin{array}{l}20,781 \\
\text { (in \%) }\end{array}$ \\
\hline Only ARAA diagnosis ${ }^{a}$ & 33 & 46 & 47 & 47 & 50 \\
\hline Continuous ARAA diagnosis & 21 & 27 & 35 & 31 & 31 \\
\hline Intermittent ARAA diagnosis ${ }^{\mathrm{a}}$ & 2 & 6 & 2 & 6 & 6 \\
\hline Omission of ARAA diagnosis & 9 & 12 & 10 & 10 & 13 \\
\hline Omission of a single diagnosis ${ }^{a}$ & 67 & 54 & 53 & 53 & 50 \\
\hline ARAA with omission of $A A$ diagnosis ${ }^{b, c}$ & 41 & 29 & 39 & 24 & 27 \\
\hline ARAA with omission of AA followed by AR & 20 & 12 & 22 & 12 & 12 \\
\hline ARAA with omission of AA followed by AR and omission & 8 & 8 & 10 & 8 & 7 \\
\hline Intermittent ARAA and AR & 11 & 8 & 6 & 4 & 7 \\
\hline Intermittent ARAA and AR followed by omission & 3 & 1 & 2 & 0 & 1 \\
\hline ARAA with omission of $A R$ diagnosis ${ }^{a}$ & 20 & 21 & 14 & 23 & 19 \\
\hline ARAA with omission of AR followed by $A^{a}$ & 13 & 9 & 8 & 11 & 9 \\
\hline ARAA with omission of AR followed by AA and omission ${ }^{b}$ & 2 & 4 & 4 & 5 & 4 \\
\hline Intermittent ARAA and AA & 5 & 7 & 2 & 7 & 6 \\
\hline Intermittent ARAA and AA followed by omission & 1 & 1 & 0 & 0 & 1 \\
\hline$A R A A, A R$ and $A A$ diagnoses ${ }^{d}$ & 6 & 4 & 0 & 7 & 4 \\
\hline ARAA with intermittent diagnoses of $A R$ and $A A$ & 4 & 3 & 0 & 5 & 3 \\
\hline $\begin{array}{l}\text { ARAA with intermittent diagnoses of AR and AA followed by } \\
\text { omission }\end{array}$ & 2 & 1 & 0 & 2 & 1 \\
\hline $\begin{array}{l}\text { AR allergic rhinitis, } A R A A \text { allergic rhinitis and allergic asthma, } \\
m S T \text { matched symptom treatment group, ST symptom treatmel } \\
\text { aSCIT vs. mST SCIT } p \leq 0.05 \\
\text { bSCIT vs. mST SCIT } p \leq 0.01 \\
\text { 'SLIT vS. mST SLIT } p \leq 0.05 \\
\text { dSLIT vs. mST SLIT } p \leq 0.01\end{array}$ & & & & & \\
\hline
\end{tabular}

diseases shows an omission of one diagnosis during the observation period (Table 4).

In the population reporting asthma diagnosis at baseline the most frequent courses of disease are omission of asthma diagnosis (30\%), continuous asthma diagnosis (29\%) and intermittent asthma diagnosis with diagnosis-free periods (13\%). Moreover $7 \%$ show occurrence of rhinitis diagnosis followed by its omission. Occurrence of a concomitant rhinitis diagnosis is reported in $28 \%$ of the asthma group but only $6 \%$ of patients document the additional rhinitis diagnosis continuously.

Due to the small number of patients with isolated asthma in the SCIT, SLIT and mST groups (SCIT $n=$ 33; $\operatorname{mST} n=66$; SLIT $n=9$; $\operatorname{mST} n=18$ ) a meaningful comparative analysis of the course of disease and medication cannot be conducted.

\section{Comparison of the course of disease between treatment strategies}

The proportion of patients with rhinitis at baseline reporting an additional asthma diagnosis during the observation period does not differ statistically significant between the SCIT group and its matched ST group (Table 3 ). In the SCIT group patients without additional asthma diagnosis are more likely to show a continuous diagnosis of rhinitis than in the mST group (odds ratio $[\mathrm{OR}]: 1.37, p=0.003$ ). They also are less likely to report intermittent courses with diagnosis-free years in the SCIT group compared to the mST group (OR: $0.63, p<0.001$ ).

The comparison of the course of disease between the SLIT group and its mST group reveals findings similar to SCIT regarding patients without asthma diagnosis. However, patients under SLIT are significantly more likely to report an additional diagnosis of asthma (OR: 1.94, $p=0.031$ ). Due to the small number of patients reporting an additional asthma diagnosis a more detailed assessment of their course of disease is not meaningful (SLIT $n=23$, ST $n=26$ ).

Omission of one diagnosis in patients with both diseases at baseline is reported for a significantly larger proportion of patients in the SCIT group compared to their mST group (OR: $1.74, p<0.001$ ) (Table 4). When one diagnosis is omitted in the SCIT group it is more likely to be the asthma diagnosis (OR: 1.36, $p=0.089$ ) and less likely to be the rhinitis diagnosis (OR: $0.65, p=0.024$ ) compared to the mST group. When no omission of a single diagnosis occurs the SCIT group shows a smaller proportion of intermittent courses compared to mST (OR: 0.39 , $p=0.035)$, whereas there is no statistically significant difference regarding remissions to diagnosis-free states. 
Similar to SCIT, patients with omission of a single diagnosis under SLIT are more likely to lose their asthma diagnosis than those in the mST group (OR: 3.57, $p=0.012$ ). Further comparisons of the course of disease between patients receiving SLIT and their mST group are not meaningful due to the small number of patients in these groups (SLIT $n=51$, ST $n=$ 102).

\section{Comparison of the course of medication between treatment strategies}

At baseline the largest proportion of rhinitis patients shows no prescription in the SCIT and SLIT groups (Table 5). In the SCIT group, rhinitis medication is more often documented than asthma medication. Within the SLIT group, rhinitis and asthma medication is prescribed for an equal proportion of patients. In both groups prescriptions for both diseases are rarely documented.

Most of the patients with both diseases hold prescriptions for asthma-related medication followed by medication for both diseases (Table 5). Only $22 \%$ of the SCIT group did not have any prescription; within the SLIT group the share was even smaller with $6 \%$. As mentioned above, the isolated asthma group is not analyzed due to the small number of observations.

During the five year observation period the average proportion of patients with both diseases receiving asthma-related prescriptions is lower in the SCIT and SLIT groups than in the mST groups (SCIT $41 \%$ vs. $46 \%$, SLIT $44 \%$ vs. $59 \%$ ) (Fig. 2). In patients with isolated rhinitis the average proportion of patients reporting asthma medication is smaller in the SCIT group compared to the mST group (12\% vs. $15 \%$ ) (Fig. 3). In both disease groups there are no relevant differences regarding the average proportion of patients receiving rhinitis medication or medication for both diseases.

In all treatment groups most of the patients with isolated rhinitis hold no disease-related prescription over the observation period (SCIT 37\%, mST 39\%; SLIT $36 \%$, mST 47\%). Among patients with both diseases the largest proportion shows continuous medication during the observation period (SCIT 25\%, mST $34 \%$, SLIT $27 \%$, mST $49 \%$ ). However, compared to $\mathrm{mST}$, rhinitis patients are less likely to receive contin- uous medication when receiving SCIT (OR: 0.75, $p=$ 0.086). Patients with both diseases in the SCIT and SLIT groups are less likely to receive continuous medication than those in the mST groups (SCIT OR: 0.65, $p=0.006$, SLIT OR 0.39, $p=0.011$ ). Furthermore, for patients with both diseases the number of medication-free years differs significantly between the treatment strategies in favor of SCIT and SLIT ( $p=0.016$, $p=0.013)$.

\section{Discussion}

The most frequently documented courses of disease are similar for patients with isolated rhinitis or isolated asthma at baseline: roughly $30 \%$ show continuous diagnosis, another $30 \%$ report omission of diagnosis and $13-20 \%$ have intermittent diagnosis and diagnosis-free periods. About $12 \%$ of rhinitis patients and $28 \%$ of asthma patients present a second allergic respiratory disease diagnosis during the observation period. In patients with both diseases there is a broader variety of courses of disease: while $30 \%$ have continuous diagnosis, $13 \%, 12 \%$ and $9 \%$ report omission of both diagnoses, omission of asthma diagnosis and omission of rhinitis diagnosis, respectively. There are also courses of omission and reoccurrence of the omitted diagnosis. In conclusion there are few courses of disease which cover a large share of patients, while the other, smaller share of patients shows a broad variety of courses of disease, sometimes changing disease group every year. This reflects the volatile nature characteristic of the course of allergic diseases.

For rhinitis patients the comparison of the course of disease between SIT and ST overall reveals larger shares of continuous and smaller shares of intermittent diagnosis in the SIT groups. Patients with both diseases in the SIT groups are more likely to report omission of one diagnosis and omission of asthma diagnosis rather than rhinitis diagnosis compared to the ST groups.

Most of the rhinitis patients have no prescribed medication over the observation period, while most of the patients with both diseases report continuous medication. The advantages regarding prescribed medication in the SIT groups compared to ST are the following: a lower average proportion of patients with asthma medication during the observation period,

Table 5 Medication groups at baseline (2007) for SCIT and SLIT

\begin{tabular}{|l|l|}
\hline Medication group & AR \\
& $\begin{array}{l}\mathrm{SCIT} \\
\text { (in \%) }\end{array}$ \\
\hline No medication & 57 \\
\hline Medication for both diseases & 7 \\
\hline Medication for asthma & 16 \\
\hline Medication for rhinitis & 20 \\
\hline
\end{tabular}

\begin{tabular}{|l|l|l|}
\hline $\begin{array}{l}\text { SLIT } \\
\text { (in \%) }\end{array}$ & $\begin{array}{l}\text { ARAA } \\
\text { SCIT } \\
\text { (in \%) }\end{array}$ & $\begin{array}{l}\text { SLIT } \\
\text { (in \%) }\end{array}$ \\
\hline 66 & 22 & 6 \\
\hline 4 & 19 & 21 \\
\hline 15 & 53 & 71 \\
\hline 15 & 6 & 4 \\
\hline
\end{tabular}

$A R$ allergic rhinitis, $A R A A$ allergic rhinitis and allergic asthma, $A A$ allergic asthma, SCIT subcutaneous immunotherapy, SLIT sublingual immunotherapy ${ }^{\text {aD }}$ Due to matching by medication group in 2007 the distribution is the same in the matched ST groups 
Fig. 2 Average proportion of patients with both diseases with disease-related prescriptions during the five year observation period (SCIT subcutaneous immunotherapy, SLIT sublingual immunotherapy, $m S T$ matched symptom treatment group)

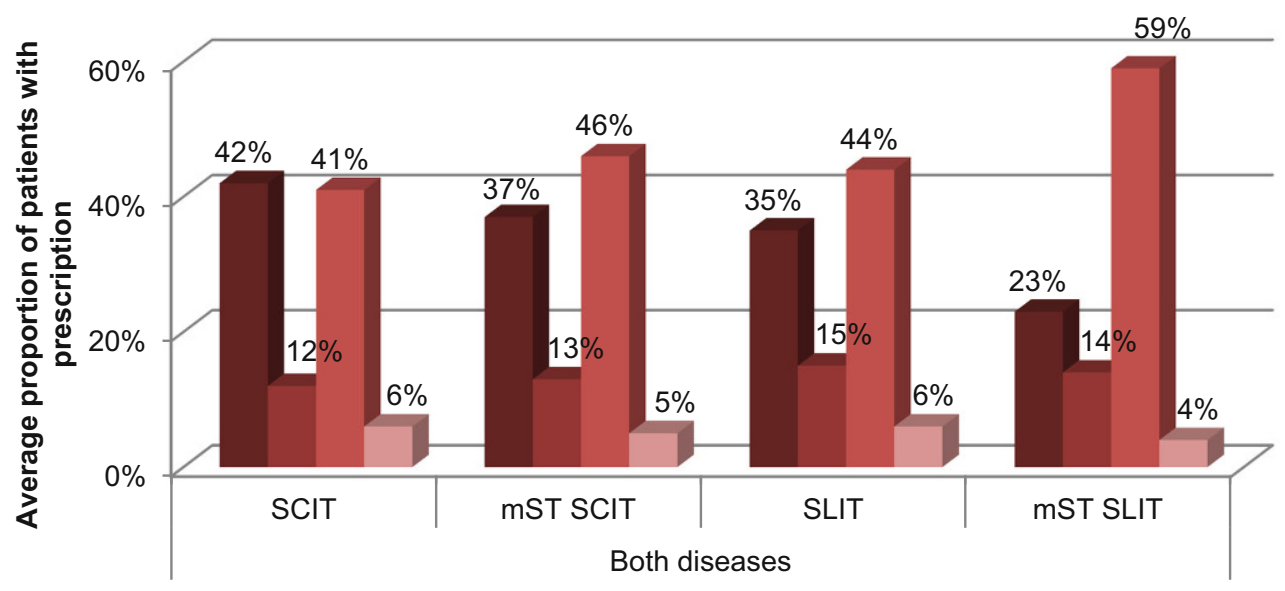

mo medication $\square$ medication for both diseases $\square$ medication for asthma ${ }^{-}$medication for rhinitis

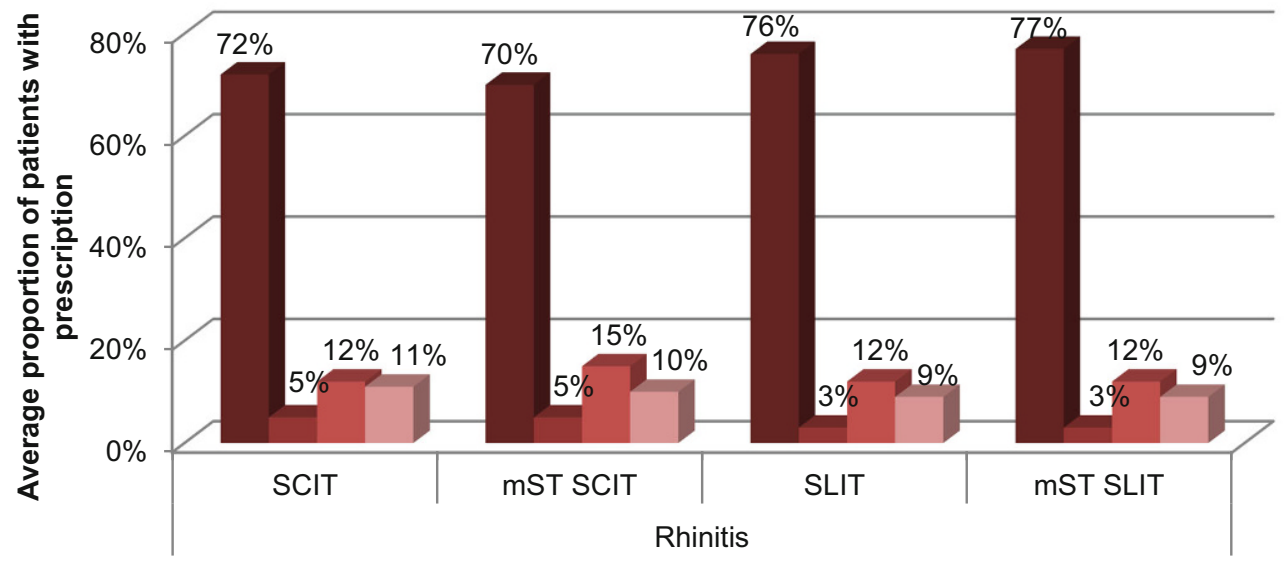

no medication $\square$ medication for both diseases $\backsim$ medication for asthma medication for rhinitis
Fig. 3 Average proportion of rhinitis patients with disease-related prescriptions during the five year observation period (SCIT subcutaneous immunotherapy, SLIT sublingual immunotherapy, mST matched symptom treatment group) ease in patients with both diseases so far. Therefore, this study adds to closing this gap of knowledge.

There are some general limitations to be considered. First, it should be noted that the presence of a disease is solely based on a diagnosis in one quarter of a year without any further validation. This broader definition of disease allows for consideration of the seasonal occurrence of symptoms as well as for the inclusion of patients treated with nonprescription drugs which is a relevant proportion of rhinitis patients.

The potential bias of this approach is assessed by additional investigation of the course of disease including only patients with disease-related prescriptions at baseline. This analysis did neither reveal relevant changes in the course of disease regarding patients receiving SCIT nor comparing the SCIT group to its mST group. Because of the small number of observations in these subgroups, analyses are not conducted for SLIT.

Second, there might be bias arising from the dependence on diagnosis documentation habits. Due to financial incentives asthma diagnoses are more likely to be documented without being clinically relevant. Therefore the reported omission rates of asthma di- 
agnoses and their difference between the SIT and ST groups should be considered as a conservative estimate. On the other hand, when only the asthma diagnosis is documented while the rhinitis diagnosis is omitted these incentives might lead to an underestimation of the proportion of patients with both diseases. Moreover, there could be over-diagnosing of any disease caused by continuation of diagnosis in the documentation systems without real need of intervention. The treatment-induced more regular physician visits in the immunotherapy groups could result in closer and more accurate documentation leading to a larger proportion of continuous diagnosis and a lower proportion of intermittent diagnosis in these groups.

Third, clinical comparability of the matched ST groups to the immunotherapy groups is limited because no information other than documented diagnoses and prescriptions were available for matching.

Fourth, for symptom treatment of rhinitis several OTC drugs are available for self-medication, which are widely used in these patients. Not including these drugs hinders comprehensive conclusions about the course of medication in allergic rhinitis patients. Furthermore omalizumab is a step five medication for asthma according to international guidelines [10] and SIT would normally not be indicated in patients treated with omalizumab. While no patients in the SLIT and their mST group are treated with omalizumab in the SCIT groups there is a skewed distribution of these patients in disfavor of SCIT (SCIT $n=$ $2,0.2 \%$; mST $n=3,0.1 \%$ ). However, due to the small proportion of affected patients this is not expected to influence the results of this analysis.

Fifth, despite the large database, the low numbers of patients with isolated asthma and of patients receiving SLIT seriously impair meaningful conclusions regarding the comparison of medication and course of disease between SIT and mST. Several exclusion criteria were inevitable for creating a definite and traceable cohort implying a loss of observations.

To assess the bias of choosing the most frequently reported treatment duration the course of disease is analyzed for patients with a plausible treatment duration not chosen for the main analysis (i.e. four years of SCIT and three years of SLIT). Apart from a slightly disadvantageous course of disease for rhinitis patients receiving four years of SCIT there are no relevant differences between the different treatment durations. Like the main analysis these comparisons are restricted by small numbers of observations in some groups (i. e. asthma and SLIT).

The therapy discontinuation rates of about $40-50 \%$ represent another reason for small number of observations in this study. There might be some cases of immunotherapy in which a new prescription is not necessary within one year. Following the definition of discontinuation in this study these cases are wrongly assigned to discontinuation. Therefore, the discontinuation rate might be slightly overestimated.

Excluding patients with immunotherapy prescriptions in the two years prior to the observation period leads to the apparently small percentage of immunotherapy prescriptions reported in this study (about 3\%). Analyzing all patients with an allergic respiratory diagnosis in 2007 ( $n=258,166)$ reveals that about $7 \%$ receive immunotherapy which is still low but comparable to the findings of other studies [24, 25]. The application rate of SIT in asthma patients $(2 \%)$ is clearly lower than that in patients with both diseases and rhinitis $(13 \%, 7 \%)$. The frequency of the SIT application forms and the baseline distribution of disease groups in this study do not differ from those reported elsewhere [24, 25].

Overall, this study provides a detailed overview of the course of treatment and the documented course of diagnoses differentiated by treatment strategy in the German SHI context. Positive effects of immunotherapy on prescribed medication over time are demonstrated, which are expected to be even stronger if nonprescribed medication were included. For patients with both diseases there is an increased likelihood of loss of asthma diagnosis when receiving immunotherapy compared to symptom treatment alone. However, lacking reliable clinical information diagnoses and prescriptions based on health insurance routine data are not sensitive enough to adequately display the benefits potentially achievable through immunotherapy.

Funding This study was funded by a grant from the German Association of Allergologists (AeDA).

Conflict of interest N. Mülleneisen, H. Renz, T. Werfel and E. Hamelmann declare that they have no competing interests. J. Biermann, J. Wasem and A.-K. Weschenfelder report grants from German Association of Allergologists (AeDA) during the conduct of the study and outside the submitted work. W. Wehrmann reports personal fees from Novartis, Galderma, ALK-Abello, Stallergens, Astellas, the German Association of Allergologists (AeDA) and Meda outside the submitted work. L. Klimek reports personal fees from ALK Abelló, MEDA, Novartis, Allergopharma, Bionorica, Boehringer Ingelheim, GSK and Lofarma as well as grants from ALK Abelló, Novartis, Allergopharma, Bionorica, GSK, Lofarma, Biomay, HAL, Leti, Roxall and Bencard outside the submitted work.

Open Access This article is distributed under the terms of the Creative Commons Attribution 4.0 International License (http://creativecommons.org/licenses/by/4.0/), which permits unrestricted use, distribution, and reproduction in any medium, provided you give appropriate credit to the original author(s) and the source, provide a link to the Creative Commons license, and indicate if changes were made.

\section{References}

1. BrozekJL, Bousquet J, Baena-Cagnani CE, Bonini S, Canonica GW, Casale TB, et al. Allergic Rhinitis and its Impact on Asthma (ARIA) guidelines: 2010 revision. J Allergy Clin Immunol. 2010;126:466-76. 
2. Bousquet J, Khaltaev N, Cruz AA, Denburg J, Fokkens WJ, Togias A, et al. Allergic Rhinitis and its Impact on Asthma (ARIA) 2008update (in collaboration with the World Health Organization, GA(2)LEN and AllerGen). Allergy. 2008;86:8-160.

3. Jarvis D, Newson R, Lotvall J, Hastan D, Tomassen P, Keil $\mathrm{T}$, et al. Asthma in adults and its association with chronic rhinosinusitis: the GA2LEN survey in Europe. Allergy. 2012;67:91-8.

4. Langen U, Schmitz R, Steppuhn H. Häufigkeit allergischer Erkrankungen in Deutschland. Ergebnisse der Studie zur Gesundheit Erwachsener in Deutschland (DEGS1). Bundesgesundheitsblatt Gesundheitsforschung Gesundheitsschutz. 2013;56:698-706.

5. Bousquet J, Knani J, Dhivert H, Richard A, Chicoye A, Ware JE Jr., et al. Quality of life in asthma. I. Internal consistency and validity of the SF-36 questionnaire. Am J Respir Crit Care Med. 1994; 149:371-5.

6. Petersen KD, Kronborg C, Gyrd-Hansen D, Dahl R, Larsen JN, Lowenstein H. Quality of life in rhinoconjunctivitis assessed with generic and disease-specific questionnaires. Allergy. 2008;63:284-91.

7. Richardson J, Khan MA, Iezzi A, Maxwell A. Comparing and explaining differences in the magnitude, content, and sensitivity of utilities predicted by the EQ-5D, SF-6D, HUI3, 15D, QWB, and AQoL-8D multiattribute utilityinstruments. MedDecis Making. 2015;35:276-91.

8. Calderon MA, Gerth van Wijk R, Eichler I, Matricardi PM, Varga EM, Kopp MV, et al. Perspectives on allergenspecific immunotherapy in childhood: an EAACI position statement. Pediatr Allergy Immunol. 2012;23:300-6.

9. Walker SM, Durham SR, Till SJ, Roberts G, Corrigan CJ, Leech SC, et al. Immunotherapy for allergic rhinitis. Clin ExpAllergy. 2011;41:1177-200.

10. Global Initative For Asthma (GINA). Global strategy for asthma management and prevention 2017. http:// ginasthma.org/2017-gina-report-global-strategy-forasthma-management-and-prevention/. Accessed 21. Apr 2017

11. World Health Organization (WHO). International classification of diseases. 2015. www.who.int.

12. Deutsches Institut für Medizinische Dokumentation und Information (DIMDI). Internationale statistische Klassifikation der Krankheiten und verwandter Gesundheitsprobleme. 10. Revision German Modification Version 2015. 2016. www.dimdi.de.

13. Pallasaho P, Juusela M, Lindqvist A, Sovijarvi A, Lundback $\mathrm{B}$, Ronmark E. Allergic rhinoconjunctivitis doubles the risk for incident asthma - results from a population study in Helsinki, Finland. Respir Med. 2011;105:1449-56.

14. de Groot EP, Nijkamp A, Duiverman EJ, Brand PLP. Allergic rhinitis is associated with poor asthma control in children with asthma. Thorax. 2012;67:582-7.

15. PfaarO, BachertC,BufeA, BuhlR,EbnerC,EngP, etal. Guideline on allergen-specific immunotherapy in IgE-mediated allergic diseases: S2kGuideline of theGermanSocietyforAl- lergology and Clinical Immunology (DGAKI), the Society for Pediatric Allergy and Environmental Medicine (GPA), the Medical Association of German Allergologists (AeDA), the Austrian Society for Allergy and Immunology (ÖGAI), the Swiss Society for Allergy and Immunology (SGAI), the German Society of Dermatology (DDG), the German Society of Oto-Rhino-Laryngology, Head and Neck Surgery (DGHNOKHC), the German Society of Pediatrics and Adolescent Medicine (DGKJ), the Society for Pediatric Pneumology (GPP), the German Respiratory Society (DGP), the German Association of ENT Surgeons (BV-HNO), the Professional Federation of Paediatricians and Youth Doctors (BVKJ), the Federal Association of Pulmonologists (BDP) and the German Dermatologists Association (BVDD). Allergo J Int. 2014;23:282-319.

16. Mahesh PA, Vedanthan PK, Holla AD, Jayaraj BS, Prabhakar AK. Time interval and the factors associated with the development of asthma in patients with allergic rhinitis. Lung. 2009; 187:393-400.

17. Makris M, Koulouris S, Koti I, Aggelides X, Sideri K, Chliva C, et al. Temporal relationship of allergic rhinitis with asthma and other co-morbidities in a Mediterranean country: a retrospective studyin a tertiary reference allergy clinic. Allergol Immunopathol (Madr). 2010;38:246-53.

18. Kurukulaaratchy RJ, Karmaus W, Raza A, Matthews S, Roberts G, Arshad SH. The influence of gender and atopy on the natural history of rhinitis in the first 18 years of life. Clin Exp Allergy. 2011;41:851-9.

19. Burgess JA, Matheson MC, Gurrin LC, Byrnes GB, Adams KS, Wharton CL, et al. Factors influencing asthma remission: a longitudinal study from childhood to middle age. Thorax. 2011;66:508-13.

20. Ekerljung L, Ronmark E, Larsson K, Sundblad B-M, Bjerg A, Ahlstedt S, et al. No further increase of incidence of asthma: incidence, remission and relapse of adult asthma in Sweden. Respir Med. 2008;102:1730-6.

21. Bronnimann S, Burrows B. A prospective study of the natural history of asthma. Remission and relapse rates. Chest. 1986;90:480-4.

22. De Marco R, Locatelli F, Cerveri I, Bugiani M, Marinoni A, Giammanco G, et al. Incidence and remission of asthma: a retrospective study on the natural history of asthma in Italy. JAllergy Clin Immunol. 2002;110:228-35.

23. Plaschke PP, Janson C, Norrman E, Bjornsson E, Ellbjar S, Jarvholm B. Onset and remission of allergic rhinitis and asthma and the relationship with atopic sensitization and smoking. Am J Respir Crit Care Med. 2000;162:920-4.

24. Biermann J, Merk H, Wehrmann W, Klimek L, Wasem J. Allergic disorders of the respiratory tract - findings from a large patient sample in the German statutory health insurance system. Allergo J. 2013;22:366-73.

25. Weschenfelder A-K, Wasem J, Biermann J. Versorgung von Patienten mit allergischer Rhinitis - eine Analyse von Routinedaten einer gesetzlichen Krankenversicherung. Allergologie. 2015;38:252-9. 